\title{
A importância da formação de professores com noções básicas de saúde: uma revisão bibliográfica
}

\author{
The importance of training teachers with basic notions of health: a bibliographic review \\ La importancia de formar docentes con nociones básicas de salud: una revisión \\ bibliográfica
}

Lázaro Saluci Ramos ${ }^{1 *}$, Irinéia Soares ${ }^{2}$, Ana Luiza de Souza Christófori², Aleksander de Souza Minguta $^{2}$, Giovani Correia Mendonça2 ${ }^{2}$, Livia França Costa $^{2}$, Gessy Moreira Reis², Eduarda de Aguiar Nunes Jordão², Luana dos Santos Rodrigues², Genivaldo dos Santos Benevides².

\section{RESUMO}

Objetivo: Propõe-se nesta revisão evidenciar como é importante formar professores para educação básica com as mínimas noções de saúde, à fim de garantir a segurança e a melhor instrução dos alunos. Revisão bibliográfica: A educação pública no Brasil se tornou o principal meio de instruir e garantir novas práticas sociais à fim do melhoramento da qualidade de vida humana. A saúde brasileira perpassa por duas vias quando se busca qualificar seu acesso, a pública e a privada. Ambas com acesso dificultado por valores financeiros ou pela baixa capacidade de atendimento. Fortalecer a instrução básica de saúde na escola é uma forma de garantir que as casas de saúde não sejam sobrecarregadas à toa, ou que em situações excepcionais, os cuidados básicos possam ser aplicados. Garantir que o docente tenha instruções básicas de saúde é uma forma de transferir ao aluno segurança e até novos conhecimentos sobre cuidados básicos. Considerações finais: $O$ estudo evidencia a necessidade de promover conhecimento em saúde em outros campos na finalidade de evitar a sobrecarga das casas de saúde brasileiras, além de garantir segurança à maior parte da população.

Palavras-chave: Serviços preventivos de saúde, Saúde, Educação.

\section{ABSTRACT}

Objective: It is proposed in this review to show how important it is to train teachers for basic education with the minimum notions of health, in order to guarantee the safety and the best instruction of students. Bibliographic review: Public education in Brazil has become the main means of instructing and guaranteeing new social practices in order to improve the quality of human life. Brazilian health goes through two ways when seeking to qualify its access, public and private. Both with access hampered by financial values or low service capacity. Strengthening basic health instruction at school is a way of ensuring that nursing homes are not overloaded for nothing, or that in exceptional situations, basic care can be applied. Ensuring that the teacher has basic health instructions is a way of transferring the student's security and even new knowledge about basic care. Final considerations: The study highlights the need to promote health knowledge in other fields in order to avoid overloading Brazilian healthcare facilities, in addition to ensuring security for most of the population.

Keywords: Preventive health services, Health, Education.

\section{RESUMEN}

Objetivo: Se propone en esta revisión mostrar cuán importante es capacitar a los maestros para la educación básica con las nociones mínimas de salud, a fin de garantizar la seguridad y la mejor instrucción de los estudiantes. Revisión bibliográfica: La educación pública en Brasil se ha convertido en el principal medio de instruir y garantizar nuevas prácticas sociales para mejorar la calidad de la vida humana. La salud brasileña pasa por dos vías cuando se busca calificar su acceso, público y privado. Ambos con acceso obstaculizado por valores financieros o baja capacidad de servicio. Fortalecer la instrucción básica de salud en la escuela es una forma de garantizar que los hogares de ancianos no se sobrecarguen por nada o que, en situaciones

1 Faculdade Multivix (MULTIVIX), Cachoeiro de Itapemirim - ES. *E-mail: lazaro321123@gmail.com

${ }^{2}$ Faculdade Vale do Cricaré (FVC), São Mateus - ES.

SUBMETIDO EM: 8/2020

ACEITO EM: 9/2020

PUBLICADO EM: 10/2020 
excepcionales, se pueda aplicar la atención básica. Asegurarse de que el maestro tenga instrucciones básicas de salud es una forma de transferir al estudiante la seguridad e incluso nuevos conocimientos sobre atención básica. Consideraciones finales: El estudio destaca la necesidad de promover el conocimiento de la salud en otros campos para evitar sobrecargar las instalaciones de salud brasileñas, además de garantizar la seguridad de la mayoría de la población.

Palabras clave: Servicios de salud preventiva, Salud, Educación.

\section{INTRODUÇÃO}

No Brasil, o histórico dos cuidados básicos com a saúde estão entrelaçados às táticas de cuidados básicos e prevenção em casos de extremos. Mesmo quando se tratam de cuidados simples e de largo conhecimento, é raro encontrar tais conhecimentos nos meios populares, sendo somente possível em pessoas com alguma proximidade ou afinidade com as áreas que compreendem a saúde. Despertar esse conhecimento nas pessoas tem a finalidade de permitir uma maior segurança à saúde do coletivo, possibilitando que nos casos em que a saúde pública faltar, esses cuidados possam ser empregados na finalidade de salvar vidas e garantir a saúde da população (CARNUT L, 2017).

Perguntas sobre condições de vida geralmente despertam prováveis novas soluções nas pessoas, como por exemplo o caminhar conjunto de saúde e educação. Não importa onde isso aconteça, seja uma escola ou uma instituição médica, a interação entre os dois é uma maneira importante de melhorar a qualidade de vida. Diante das necessidades das escolas, a construção de práticas de ensino relacionadas a essa interação é um enorme desafio. É necessário discutir os conceitos que sustentam ações saudáveis como prática de ensino e fazer com que todos os participantes compreendam claramente esses conceitos. $O$ conceito de pedagogia é entender, teorizar e praticar diferentes formas de educação e expressar a maneira de operar e executar comportamentos educacionais (CARVALHO FFB, 2015).

$\mathrm{Na}$ década de 90 , floresceram as pesquisas brasileiras sobre ensino e saúde. A primeira pesquisa realizada na época focava o cultivo do conhecimento em saúde para professoras e professores levando em consideração várias questões, vários investimentos foram feitos e diferentes teorias, conceitos e métodos metodológicos foram adotados.

Esses estudos inaugurais marcam a tradição da pesquisa nesse campo e, ainda hoje são a espinha dorsal da geração de conhecimento sobre o assunto. Alguns desses grupos reformularam seu foco, reduzindo o investimento em análises de trabalho e saúde, enquanto outros mantiveram atividades de pesquisa em andamento. São utilizados métodos qualitativos e quantitativos, embora sejam conduzidos por grupos diferentes, juntos oferecem amplas possibilidades de debate e complementaridade, pois possuem algo em comum, mesmo que não tenham sido elaborados entre esses estudos antes (ARAÚJO TM, et al., 2019).

Devido à Lei de Diretrizes e Bases da Educação Nacional (LDB) e sua determinação em promover os currículos educacionais a substituir o currículo mínimo e a institucionalização de novas políticas de saúde, a educação em saúde sofreu grandes mudanças na última década. Portanto, é evidente que a formação de professores com noções básicas de saúde no ensino superior ainda é experimental e possui um profundo entendimento da naturalização da prática docente. A prática profissional reconhecida é considerada o padrão da capacidade do professor acima mencionado. Nesse caso, a imagem ou modelo de excelentes professores aparecerá na imaginação de alunos e professores ao mesmo tempo, dependendo do campo, profissão e experiência e experiência teórica de alunos e professores (MENEGAZ JC, et al., 2013).

Políticas e programas de saúde pública e educação são essenciais para o treinamento do cidadão e a melhoria da qualidade de vida e saúde das pessoas. Um melhor nível de educação está relacionado às pessoas mais saudáveis, assim como as pessoas saudáveis têm maior probabilidade de adquirir conhecimentos e conhecimentos adequados da educação formal e não formal. Além de transmitir conhecimento sobre saúde, as escolas também são organizadas em disciplinas. No processo de aquisição de habilidades que apoiam a aprendizagem ao longo da vida, as escolas também devem educar e desenvolver valores e atitudes críticas relacionadas às realidades e estilos de vida sociais e apoiar 0 empoderamento e a autonomia da promoção da saúde (LOPES IE, et al., 2018). 
O objetivo desse artigo é expor a necessidade de inserir professores com noções básicas de saúde, à fim de transmitir o conhecimento e promover uma sociedade mais conhecedora com noções básicas de primeiros socorros e saúde em geral. É público e notório que os sistemas de saúde brasileiros não atendem a demanda disposta, e por isso é preciso garantir que a população conheça técnicas básicas de saúde para garantir o mínimo de compreensão, sendo assim, a escola que é uma casa que dissemina o direito básico à educação, à partir do momento em que transmite esse conhecimento, amplia seus objetivos e sua incisão positiva na sociedade.

\section{REVISÃO BIBLIOGRÁFICA}

A educação em saúde é um dos principais meios de promover a atenção primária à saúde no Brasil. Reconhecer que a saúde possui características multidimensionais e que os usuários são sujeitos à educação buscando autonomia é condição necessária para a inserção de conhecimentos nesse campo. Essa premissa satisfaz a discussão sobre promoção da saúde que se intensifica no Brasil desde a década de 1980, com a convocação da Conferência Internacional de Promoção da Saúde, que define como princípio a multicausalidade dos processos saúde-doença, participação intersetorial, social e sustentabilidade. A mudança na educação em saúde nessa direção é recente.

Desde a década de 1970, os usuários participam do processo educacional como órgão principal e, na década de 90, começaram a considerar a psicologia social e a subjetividade do indivíduo (CARNEIRO ACLL, et al., 2012).

Os diferentes conceitos de promoção da saúde podem ser divididos em duas categorias: uma é comportamental e a outra, relacionada à qualidade de vida. Em primeiro lugar, a promoção da saúde inclui atividades que visam mudar o comportamento dos sujeitos, com foco no conteúdo educacional, que envolve principalmente conteúdos educacionais relacionados aos riscos comportamentais que podem ser alterados. O segundo é baseado no seguinte entendimento: saúde é o produto de múltiplos fatores relacionados à qualidade de vida, incluindo padrões adequados de alimentação, nutrição, moradia e higiene, trabalho e importa receber educação durante todo o ciclo de vida sobre um ambiente físico limpo, interação social, estilo de vida responsável e assistência médica adequada (SILVA JP, et al., 2018).

Primeiros socorros (PS) é definido como atendimento imediato às vítimas de acidentes ou doenças súbitas quando suas condições físicas ameaçam suas vidas. Nesse caso, os socorristas devem manter funções importantes e evitar a piora da condição do paciente até a chegada de assistência especializada. O primeiro serviço pode ser realizado por qualquer pessoal treinado e não é uma ação privada de um profissional de saúde. Embora a literatura tenha difundido a ideia de que o conhecimento e o ensino sobre PS são limitados a esses profissionais ou profissionais das universidades (SILVA DP, et al., 2018).

Ainda para Silva DP; et al. (2018); no Brasil, os acidentes são a principal causa de morte de crianças entre 1 e 14 anos. No ambiente escolar, esses incidentes sempre atraíram a atenção das pessoas, pois estão sendo cada vez mais recorrentes, e mais ainda em creches e pré-escolas com crianças de 0 e 6 anos. Nesse caso, o ambiente escolar torna-se um local de alerta precoce para emergências. Os alunos podem se tornar vítimas e os docentes possíveis testemunhas para esses eventos, o que os torna os principais participantes para intervir nessas situações, reduzindo a complexidade e melhorando portanto, o ambiente escolar, principalmente da perspectiva dos professores, o ensino e a popularização da prática de PS que são de fundamental importância.

Reconhecer o vínculo entre saúde e educação não é novo. Sob o argumento dessa estreita conexão entre os dois campos, há pelo menos um consenso: um bom nível de educação está relacionado a uma população mais saudável e uma população saudável é mais provável que obtenha conhecimento da educação formal e não formal. De acordo com o local em que você fala e a escrita usada, você pode encontrar os mais diferentes discursos e cenários, ou seja, sob tal argumento, você pode encontrar o método mais adequado para o assunto. Na América Latina, caracterizada por desigualdade histórica e embaraçosa, dados oficiais mostram que, apesar dos grandes desafios, os indicadores sociais e de saúde melhoraram, isso se deu ao crescimento da instrução em saúde, como por exemplo, a ampliação das grades das disciplinas relacionadas ao campo (CASEMIRO JP, et al., 2014). 
Na década de 1960, movimentos sociais como o movimento de massas liderado pelo educador Paulo Freire influenciaram o campo da prática de educação em saúde, absorvendo a participação e o conhecimento das pessoas da região, resultando em um processo educacional mais democrático. Essa influência se reflete na Campanha Universal de Educação em Saúde, formada nos últimos 40 anos, formada pela reflexão, produção de conhecimento e ativismo de múltiplas organizações (como a Associação Nacional de Educação em Saúde) criada durante esse período.

A rede de educação em massa e saúde surgiu de uma conexão das campanhas e práticas nacionais de educação em massa e saúde. A educação em saúde pública é um movimento histórico de mudança, originalmente proposto por profissionais médicos que estavam insatisfeitos com a comercialização e práticas repetitivas de serviços médicos que não atendiam às camadas mais carentes da sociedade brasileira (FALKENBERG MB, et al., 2014).

\section{Benefícios de um corpo docente com conhecimentos básicos de saúde}

Um corpo docente com conhecimentos básicos de saúde pode transmitir uma infinidade de benefícios ao corpo discente. Desde técnicas de primeiros socorros, como atuar em necessidades emergenciais em acidentes com alunos, isso em especial na educação infantil, onde é comum acidentes envolvendo crianças, como transmitir noções básicas de cuidados com o próprio corpo, como higiene pessoal, cuidados com a saúde mental, reconhecer quando um aluno está ou não gozando da plenitude das suas capacidades para desfrutar do ambiente escolar. Esses pontos devem ser observados sempre, e quase nunca é possível devido à falta de conhecimento específico (RAPOSO IPA e GONÇALVES MBC, 2018).

A relação entre os setores de educação e saúde tem muitas semelhanças no campo das políticas públicas, porque se baseiam em direitos básicos universais e, portanto, favorecem a aproximação dos cidadãos nos diferentes cantos do país. Historicamente, a afinidade é uma unidade, pelo menos no Brasil, na década de 1950, quando o Ministério da Educação e Saúde (MES) foi dividido em dois departamentos: o Ministério da Saúde e o Ministério da Educação, com autonomia institucional e direito de formular e implementar políticas em seu campo. Nesse caso, as ações formuladas pelo Ministério da Saúde nacional do MES original passaram a ser de responsabilidade do Ministério da Saúde (BRASIL, 2009).

A educação em saúde é um meio de melhorar a qualidade de vida, expressando ciência e conhecimento popular, instrumentalizando os não profissionais e promovendo seus pensamentos e práticas em sua vida cotidiana para atender às suas reais necessidades. Os Primeiros Socorros (PS)desempenham um papel necessário, pois a falta de conhecimento pode levar a muitos problemas, como tratamento incorreto das vítimas e, às vezes, solicitações desnecessárias de assistência profissional. A importância da educação em saúde no PS é que as vítimas que precisam de cuidados imediatos precisam ser atendidas e é necessária assistência rápida e de alta qualidade. Entende-se que as ações tomadas no local do incidente ajudarão a sobrevivência das vítimas (SILVA DP, et al., 2018).

Mesmo que as reformas educacionais tenham redirecionado a produção de cursos flexíveis e sensíveis para prioridades regionais e contextos específicos de saúde, o treinamento acadêmico em saúde mental ainda tem limitações, sendo as mais importantes a redução da carga de trabalho, a redução do estresse e os métodos de ensino das universidades instrumentais e como elas afetaram o desenvolvimento insuficiente de táticas e habilidades para esse tipo de atendimento.

No entanto, o foco da prática de saúde na doença fortaleceu o método do biólogo de preferir o tratamento medicamentoso, resultando em um treinamento fragmentado sem fim, o que é inconsistente com o princípio holístico de enfermagem dos profissionais de saúde. Observando a ineficiência do tratamento medicamentoso e muitos casos, e a falta de inclusão do aluno submetido a estes tratamentos, faz-se necessário provir profissionais ambientados com as necessidades desses alunos (RODRIGUES J, et al., 2019).

A medicalização é entendida como um processo que envolve dor psicológica, de origem social, política e / ou escolar, e é interpretada como uma questão de origem biológica e médica. Um aluno que exibe comportamento anormal, não segue regras aceitáveis e socialmente construídas, tem dificuldades de aprendizado, tem um ritmo diferente de seus colegas ou exibe um comportamento que não é adequado para 
a escola é geralmente direcionado ao serviço médico e, aos olhos da medicina, ele é considerado o indivíduo mórbido e responsável por suas próprias dificuldades, sem considerar questões sociais, como baixa qualidade do ensino e políticas públicas insuficientes para efetivamente estabelecer o direito à educação (BENEDETTI MD, et al., 2018).

O Brasil ainda é um país tropical com instalações sanitárias precárias, deixando os indivíduos desprotegidos e vulneráveis porque estão mais expostos a larvas e ovos de parasitas. Nesse caso, no território brasileiro se torna um quintal amplo na propagação de doenças, muitas vezes devido à falta de orientação do aluno que é o agente a ser preparado para a sociedade, o número de pessoas afetadas por parasitas ambientais e falta de saneamento é mais grave. Tudo isso pode ser potencializado pela ausência de conhecimentos básicos de saúde.

Destaca-se, portanto, a importância da educação voltada para a saúde, que envolve todo o ambiente escolar e, nesse sentido, é a atividade cotidiana de educadores e estudantes de todos os níveis educacionais, portanto, a educação em saúde parece ser uma prática. Esse programa educacional visa induzir as pessoas a desenvolver hábitos que promovem a saúde e previnem doenças (SILVA ACO, 2015).

De todos os problemas escolares envolvendo saúde, a resolução passa pela necessidade de instrução e ampliação da grade de conhecimentos. O alunado não consegue desvendar certas situações que não são tratadas no seu cotidiano, o que facilitaria se fosse.

O professor quando perguntado, mesmo quando o assunto não diz respeito a disciplina em questão, está fadado à pelo menos indicar possíveis fontes de pesquisa, pois este é colocado em situação de transmissor do conhecimento, e é dado ao aluno a referência do docente para sanar a ausência do seu conhecimento.

E torna-se de ampla valia, incentivar às práticas de saúde na escola e a educação em saúde para futuros profissionais da educação, na finalidade de garantir um ambiente escolar mais seguro e instrutivo (PAES CCDC e PAIXÃO ANP, 2016).

\section{As políticas públicas de educação em saúde}

Os direitos garantidos pela Constituição e o estabelecimento do Sistema Único de Saúde (SUS) nas décadas seguintes promoveram a descentralização do poder político e administrativo, participação social, mudanças nos modelos médicos, aumento do acesso aos serviços públicos e melhoria dos indicadores de saúde. A implementação do SUS encontrou alguns obstáculos.

Nos anos 90, o domínio da política econômica e a agenda nacional de reformas inspiradas no neoliberalismo impuseram restrições ao desenvolvimento da lógica de segurança e das políticas sociais gerais.

O aumento do poder do governo de esquerda desde 2003 despertou expectativas de que um modelo de desenvolvimento redistribuído foi implantado, mas vários problemas do sistema de saúde não foram resolvidos (MACHADO CV, et al., 2017).

A Política Nacional de Educação Permanente em Saúde (PNEPS) foi formulada em 2004 e é um marco na educação e no trabalho em saúde do país. As lutas e esforços dos defensores focados na educação dos profissionais de saúde são o resultado de esforços para promover a transformação dos métodos de trabalho em saúde.

O PNEPS é no Brasil uma conquista da sociedade. Criou o PNEPS como uma estratégia de treinamento e desenvolvimento para os trabalhadores do SUS, cuja estrutura legal é o Regulamento GM/MS nํ198, em 13 de fevereiro de 2004.

Suas diretrizes de implementação foram subsequentemente emitidas pelo Regulamento GM / MS no 1.996, que era 20 de agosto de 2007. Este é um passo importante na promoção de ações de políticas regionais e participação interinstitucional por meio das Comissões de Integração Ensino-Serviço (CIES). Além disso, foram estabelecidos orçamentos para projetos e ações para uma alocação transparente e clara (BRASIL, 2018). 
A portaria dispõe: Art. $1^{\circ}$ Definir novas diretrizes e estratégias para a implementação da Política Nacional de Educação Permanente em Saúde, adequando-a às diretrizes operacionais e ao regulamento do Pacto pela Saúde. Parágrafo único. A Política Nacional de Educação Permanente em Saúde deve considerar as especificidades regionais, a superação das desigualdades regionais, as necessidades de formação e desenvolvimento para o trabalho em saúde e a capacidade já instalada de oferta institucional de ações formais de educação na saúde. Art. $2^{\circ} \mathrm{A}$ condução regional da Política Nacional de Educação Permanente em Saúde dar-se-á por meio dos Colegiados de Gestão Regional, com a participação das Comissões Permanentes de Integração Ensino-Serviço (BRASIL, 2007).

Nos últimos anos, no Brasil, muitas iniciativas e experiências para avaliar a atenção primária foram implementadas para melhorar as políticas de saúde. O Plano Nacional de Melhoria dos Serviços e Qualidade da Atenção Primária à Saúde (PMAQ), tem como principal objetivo estimular o acesso e a qualidade dos serviços de atenção primária à saúde, para que as ações governamentais em atenção à saúde tenham maior transparência e eficácia (MACHADO MFAS, et al., 2015).

Dentre as diversas ações de promoção da saúde realizadas na atenção básica, o PMAQ avaliou o "Plano de Saúde Escolar". Para o Ministério da Saúde, o "Plano de Saúde Escolar" visa proporcionar uma série de ações de prevenção, promoção e saúde para crianças, adolescentes e jovens, com o apoio da educação básica pública através do Programa Saúde na Escola (PSE). Isso se dá devido a comunicação clara entre a escola pública e a equipe da Estratégia Saúde da Família (ESF) por meio de ações voltadas aos alunos (MACHADO MFAS, et al., 2015).

Além da grande gleba de políticas públicas direcionadas a saúde na escola e a educação em saúde, há que considerar que a instrução social facilitaria a disseminação e a efetivação dessas políticas. O professor quando transmite, didaticamente, matéria sobre saúde e cuidados básicos, abre portas aos alunos e garante o conhecimento sobre a área em questão. Conhecer torna-se o primeiro passo para o aprender, e posteriormente, o colocar em prática. Para tal, o professor necessita de um saber específico, sendo assim, tendo que receber instruções corretas para transmitir como matéria aos alunos. Bastaria incluir na grade dos cursos superiores de formação de professores, disciplinas relacionadas a temática, e para os professores já formados, políticas de formação continuada com noções básicas de saúde (ATALIBA P e MOURÃO L, 2018).

Para Bomfim Ml, et al. (2014); deve-se enfatizar o potencial de formação de professores no campo da educação profissional no âmbito do SUS, em vez de se capacitar para resolver os problemas de saúde do país ou ainda definir as condições para que os trabalhadores mantenham a saúde para atender à demanda do mercado. Portanto, a ideia é defender a educação continuada, com foco na leitura do mundo e no desempenho crítico, por isso é diferente do processo de formação da ordem social existente, em que os professores não estão em um estado de tensão, mas em um estado de adaptação.

\section{CONSIDERAÇÕES FINAIS}

Conclui-se que, a formação de professores com noções básicas e até mesmo mínimas de saúde, é de importância singular no enriquecimento do conhecimento transmitido, como também na proteção e segurança dos alunos. $O$ aluno que descobre o universo da saúde e os cuidados básicos a serem tomados a partir de práticas de primeiros socorros, por exemplo, fazem parte de uma sociedade mais comunicativa e proativa. Os programas de educação em saúde, apesar de serem uma inovação do século XXI, precisam ser fortalecidos e ampliados através de novas políticas, que complementem ou favoreçam com novas propostas os projetos já existentes.

\section{REFERÊNCIAS}

1. ARAÚJO TM, et al. Trabalho e saúde de professoras e professores no Brasil: reflexões sobre trajetórias das investigações, avanços e desafios. Cadernos de Saúde Pública, 2019; 35(1): 1-16.

2. ATALIBA P, MOURÃ̃ L. Avaliação de impacto do Programa Saúde nas Escolas. Psicologia Escolar e Educacional, 2018; 22(1): 27-36. 
3. BENEDETTI MD, et al. Medicalização e educação: análise de processos de atendimento em queixa escolar. Psicologia Escolar e Educacional, 2018; 22(1): 73-81.

4. BOMFIM MI, et al. Formação docente na área da saúde: avaliação, questões e tensões. Interface (Comunicação Saúde Educação), 2014; 18(51): 749-758.

5. BRASIL. 2007. In: PORTARIA № 1.996, de 20 de agosto de 2007, Dispõe sobre as diretrizes para a implementação da Política Nacional de Educação Permanente em Saúde. Brasília: Ministério da Saúde. Disponível em: http://bvsms.saude.gov.br/bvs/saudelegis/gm/2007/prt1996_20_08_2007.html. Acesso em: 19 de jul. 2020.

6. BRASIL. 2009. In: CADERNOS de atenção básica: saúde na escola. Brasília: Ministério da Saúde. Disponível em: https://bvsms.saude.gov.br/bvs/publicacoes/cadernos_atencao_basica_24.pdf. Acesso em 20 de jul. 2020.

7. BRASIL. 2018. In: POLÍTICA Nacional de Educação Permanente em Saúde: Secretaria de Gestão do Trabalho e da Educação na Saúde, Departamento de Gestão da Educação na Saúde. Brasília: Ministério da Saúde. Disponível em: https://bvsms.saude.gov.br/bvs/publicacoes/politica_nacional_educacao_permanente_saude_fortalecimento.pdf. Acesso em: 19 de jul. 2020.

8. CARNEIRO ACLL, et al. Educação para a promoção da saúde no contexto da atenção primária. Rev Panam Salud Publica, 2012; 31(2): 115-120.

9. CARNUT L. Cuidado, integralidade e atenção primária: articulação essencial para refletir sobre o setor saúde no Brasil. Saúde debate, 2017; 41(115): 1177-1186.

10. CARVALHO FFB. A saúde vai à escola: a promoção da saúde em práticas pedagógicas. Physis Revista de Saúde Coletiva, 2015; 25(4): 1207-1227.

11. CASEMIRO JP, et al. Promover saúde na escola: reflexões a partir de uma revisão sobre saúde escolar na América Latina. Ciência \& Saúde Coletiva, 2014; 19(3): 829-840.

12. FALKENBERG MB, et al. Educação em saúde e educação na saúde: conceitos e implicações para a saúde coletiva. Ciência \& Saúde Coletiva, 2014; 19(3): 847-852.

13. LOPES IE, et al. Eixos de ação do Programa Saúde na Escola e Promoção da Saúde: revisão integrativa. Saúde e debate, 2018; 42(118): 773-789.

14. MACHADO CV, et al. Políticas de saúde no Brasil em tempos contraditórios: caminhos e tropeços na construção de um sistema universal. Cad. Saúde Pública, 2017; 33(2): 144-161.

15. MACHADO MFAS, et al. Programa saúde na escola: estratégia promotora de saúde na atenção básica no Brasil. JournalofHumanGrowthandDevelopment, 2015; 25(3): 307-312.

16. MENEGAZ JC, et al. O bom professor na área da saúde: uma revisão integrativa da literatura. Saúde \& Transformação Social, 2013; 4(4): 92-99.

17. PAES CCDC, PAIXÃO ANP. A importância da abordagem da educação em saúde: revisão de literatura. REVASF, $2016 ; 6(11): 80-90$.

18. RAPOSO IPA, GONÇALVES MBC. A Saúde dos Amigos de Sala de Aula Interfere no Desempenho Escolar do Aluno?. Estudos Econômicos (São Paulo), 2018; 48(2): 311-337.

19. RODRIGUES J, et al. Ensino da saúde mental em enfermagem na percepção dos professores. Texto \& Contexto Enfermagem, 2019; 28(1): 1-12.

20. SILVA ACO. Educação para saúde: propostas de ensino incentivando hábitos saudáveis. Monografia (curso de Ciências Biológicas). Universidade Estadual da Paraíba, Campina Grande, 2015; 50 p.

21. SILVA DP, et al. Primeiros socorros: objetivo de educação em saúde para professores. Revenferm UFPE online, 2018; 12(5): 1444-1453.

22. SILVA JP, et al. Promoção da saúde na educação básica: percepções dos alunos de licenciatura em enfermagem. Revista Gaúcha de Enfermagem, 2018; 39(1): 1-11. 